Article

\title{
Preparation and Properties of Self-Healing and Self-Lubricating Epoxy Coatings with Polyurethane Microcapsules Containing Bifunctional Linseed Oil
}

\author{
Haijuan Yang ${ }^{1,2}$, Qiufeng Mo ${ }^{1,2}$, Weizhou Li ${ }^{1,2, *}$ and Fengmei Gu ${ }^{1,2}$ \\ 1 School of Resources, Environment and Materials, Guangxi University, Nanning 530004, China; \\ yanghaijuan2019@163.com (H.Y.); 18078131362@163.com (Q.M.); gufengmei32@163.com (F.G.) \\ 2 Guangxi Key Laboratory of Processing for Non-ferrous Metals and Featured Materials, Guangxi University, \\ Nanning 530004, China \\ * Correspondence: wz-Li@hotmail.com; Tel.: +86-1507-716-5102
}

Received: 31 July 2019; Accepted: 24 September 2019; Published: 27 September 2019

\begin{abstract}
An organic coating is commonly used to protect metal from corrosion, but it is prone to failure due to microcracks generated by internal stress and external mechanical action. The self-healing and self-lubricating achieved in the coating is novel, which allows an extension of life by providing resistance to damage and repair after damage. In this study, a new approach to microencapsulating bifunctional linseed oil with polyurethane shell by interfacial polymerization. Moreover, the self-healing and self-lubricating coatings with different concentrations of microcapsules were developed. The well-dispersed microcapsules showed a regular spherical morphology with an average diameter of $\sim 64.9 \mu \mathrm{m}$ and a core content of $74.0 \mathrm{wt} . \%$. The results of the salt spray test demonstrated that coatings containing microcapsules still possess anticorrosion, which is improved with the increase of microcapsules content, after being scratched. The results of electrochemical impedance spectroscopy showed a $|\mathrm{Z}|_{\mathrm{f}=0.01 \mathrm{~Hz}}$ value of $10^{4} \Omega \cdot \mathrm{cm}^{2}$ for pure epoxy coating after being immersed for 3 days, whereas the coating with $20 \mathrm{wt}$ \% microcapsules was the highest, $10^{10} \Omega \cdot \mathrm{cm}^{2}$. The results of friction wear showed that the tribological performance of the coating was enhanced greatly as microcapsule concentration reached $10 \mathrm{wt}$ \% or more, which showed a $86.8 \%$ or more reduction in the friction coefficient compared to the pure epoxy coating. These results indicated that the coatings containing microcapsules exhibited excellent self-healing and self-lubricating properties, which are positively correlated with microcapsules content.
\end{abstract}

Keywords: coating; microcapsules; linseed oil; self-healing; self-lubricating

\section{Introduction}

Corrosion causes huge economic losses every year in various fields, such as marine, construction, and aerospace [1,2]. Organic coating can block the migration passage of water and oxygen to the metal surfaces, and it is commonly used to protect metal from corrosion [3,4]. Nonetheless, during its preparation and use, the coating is prone to microcracks inside the structure due to solvent evaporation, mechanical action, temperature change, etc. $[1,5,6]$. As the crack expands, the metal is contacted with the corrosive medium and the coating loses its protective ability. Furthermore, the initial microcracks are very difficult to detect and repair. So, the development of alternative protections is extremely urgent.

Self-healing coatings have been considered as a promising method to address the above challenges, due to the automatic damages healing without external intervention [7,8]. White et al. [9] first encased dicyclopentadiene (DCPD) inside urea-formaldehyde (UF) shells via in situ polymerization to form microcapsules, and found that the epoxy matrix containing microcapsules and Grubbs catalyst showed self-healing after damage. After that, loading microcapsules with healing agents to develop self-healing 
coating has attracted great interest [10,11]. In addition to DCPD, various healing agents have been microencapsulated for self-healing systems, including epoxy [12-14], polydimethylsiloxane [15-17], dicyclohexylmethane diisocyanate (HMDI) [18], hexamethylene diisocyanate (HDI) [19], isophorone diisocyanate (IPDI) [20-23], Polyaryl polymethylene isocyanates (PAPI) [24], tung oil (TO) [25], linseed oil (LO) [6], etc. Among them, LO is a good candidate for healing agents because it is cheap, environmentally friendly, and air-drying, which can be polymerized with oxygen into a flexible and water-repellent coating [26-28]. Moreover, the lubricity of LO has been reported. Kozdrach et al. [29] have shown the impact of polyvinylpyrrolidone on friction and wear performances of LO-based grease. LO has a similar viscosity and thermal stability to lubricate oil, which make it a prominent lubricant. This means tLO can act as a bifunctional agent for self-healing and self-lubricating. Poly(urea-formaldehyde) microcapsules containing TO were studied to heal and resist wear [30]. HMDI-filled microcapsules has been reported as the healing and lubricating agent [18]. The self-healing and self-lubricating achieved in the coating is novel, which allows an extension of life by providing resistance to damage and repair after damage. So far, most studies have concentrated on single-function coatings, whereas dual-function coatings with self-healing and self-lubricating have rarely been reported [18,30,31]. The multifunctional characteristics achieved in the coating matrix are the focus of smart coatings in the future. Developing smart coatings on self-healing and self-lubricating properties is extremely challenging [18]. Therefore, LO was selected as the core material for this study.

Suryanarayana et al. [6] encased LO in urea formaldehyde (UF) shells through in situ polymerization, and found that $\mathrm{LO}$ could heal the coating to prevent metal corrosion. Szabó et al. [32] loaded LO, co-octoate, and octadecylamine (ODA) within UF microcapsules to recover damage and obtain a better anticorrosion ability. Hasanzadeh et al. [5] applied UF to encase $\mathrm{LO}$ and $\mathrm{CeO}_{2}$ nanoparticles, investigating the self-healing ability of coatings with these microcapsules. UF is commonly used to encapsulate LO via in situ polymerization in most studies. UF resin is generally formed by reacting urea with formaldehyde in a stoichiometric ratio. Moreover, UF resin may be degraded to release formaldehyde if it is affected by temperature, humidity, $\mathrm{pH}$ and chemicals during use [33]. It is accompanied by safety hazards during use. It is urgent to develop ecofriendly shell materials to load LO microcapsules. Therefore, polyurethane was used as the shell material of the microcapsules in this study.

In this study, we encapsulated LO into polyurethane shells instead of unfriendly UF shells. The polyurethane shell was formed by interfacial polymerization using TMP and polyisocyanate. The prepared LO-loaded microcapsules were embedded into epoxy coating for investigation of their self-healing and self-lubricating properties. Scanning electron microscopy (SEM), Fourier transform infrared spectroscopy (FTIR), and thermogravimetric analysis (TGA) were used to characterize the properties of the microcapsules. Salt spray tests, electrochemical impedance spectroscopy (EIS), and friction wear test were carried to evaluate the coating on the self-healing and self-lubricating properties.

\section{Materials and Methods}

\subsection{Materials}

Epoxy resin Epikoe 862, thinner Heloxy 8, and related hardener Epikure 205 were obtained from Hexion Specialty Chemicals. (Columbus OH, America). LO serves as the core material and was procured from Damao Chemical Reagent Factory (Tianjin, China). Desmodur L-75 with an isocyanate group (NCO) content of $13.3 \pm 0.4 \mathrm{wt} . \%$, used as a material for preparing the shell, was purchased from Bayer MaterialScience (Leverkusen, Germany). 1,1,1-trimethylolpropane (TMP) and gum arabic (GA) were purchased from Aladdin (Shanghai, China). Ethyl acetate (EtOAc) was used as solvent and obtained from Guangdong Guanghua Sci-Tech Co.,Ltd. (Guangdong, China) The above chemicals were utilized without further purification. 


\subsection{Synthesis of PU-Encapsulated LO Microcapsules}

Polyurethane (PU) microcapsules containing LO were prepared in two steps by interfacial polymerization. In the first step, an organic phase was carried out by dissolving $\mathrm{LO}(24.0 \mathrm{~g})$ and L-75 (8.0 g) into EtOAc (20.0 g). A mixture was prepared by mixing the organic phase and aqueous emulsifier solution, which was provided by dissolving GA $(12.0 \mathrm{~g})$ into $120.0 \mathrm{~g}$ of deionized water. The mixture was emulsified at $1200 \mathrm{rpm}$ for $5 \mathrm{~min}$ to acquire stable emulsion system. In the second step, polymerization reaction was started by adding a solution containing $4.0 \mathrm{~g}$ of TMP and $20.0 \mathrm{~g}$ deionized water to the emulsion system dropwise. The reaction system was heated to $50{ }^{\circ} \mathrm{C}$ for $1.5 \mathrm{~h}$ with continuously stirring at $400 \mathrm{rpm}$ to complete the formation of the microcapsules. The suspended microcapsules were separated after placing the reacted solution for $2 \mathrm{~h}$. The microcapsules were filtered under negative pressure with deionized water for several times and then dried in the environment for $24 \mathrm{~h}$.

\subsection{Preparation of Epoxy Composites}

Epoxy resin was obtained by formulating Epikoe 862, Epikure F 205, and Heloxy 8 at mass ratio of 1:0.58:0.1. Microcapsules were added in the epoxy resin at different concentrations is 5, 10, 15, and $20 \mathrm{wt} . \%$. The pure epoxy sample without microcapsules was prepared as a control sample.

The samples for salt spray test and EIS measurements were prepared via applying a coating having a dry thickness of $250 \pm 20 \mu \mathrm{m}$ on steel plate $\left(120 \times 50 \times 1 \mathrm{~mm}^{3}\right)$ with a drawdown bar. After all samples were cured at room temperature for $24 \mathrm{~h}$, cross scratches deep into the substrate were created on samples using a homemade scratch apparatus (Figure 1). Scratched samples were placed at room temperature for 5 days and then subjected to thermal curing at $80^{\circ} \mathrm{C}$ for $4 \mathrm{~h}$ to ensure the completion of self-healing [34], followed by salt spray and EIS tests.

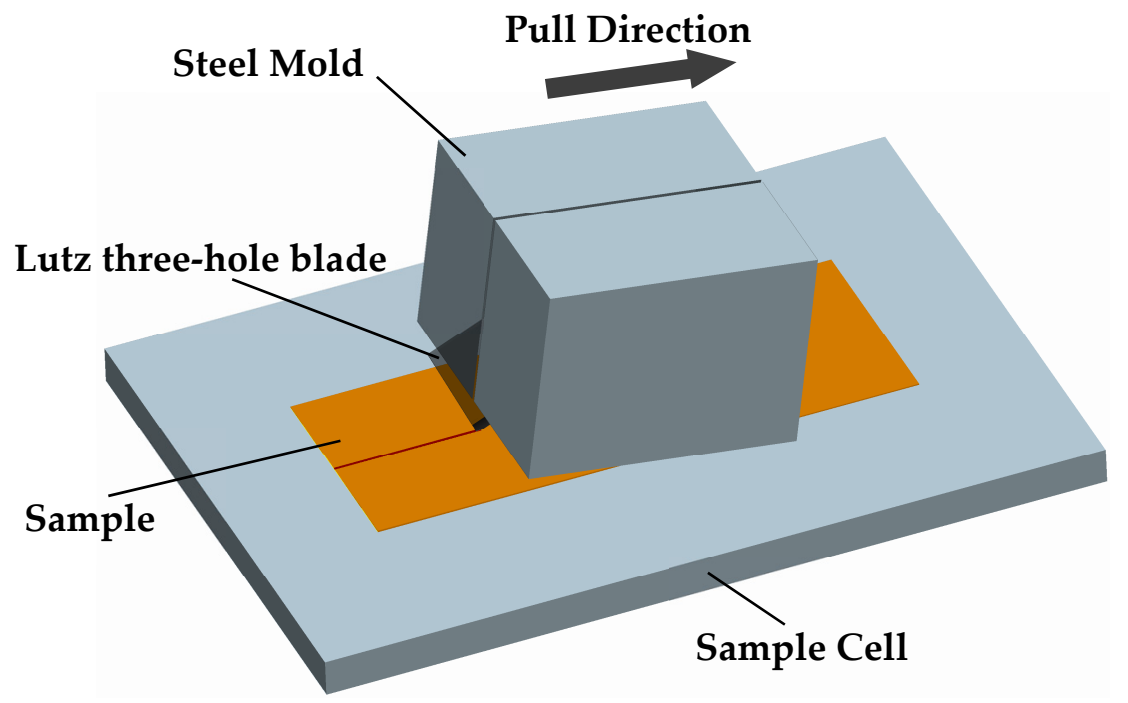

Figure 1. Schematic diagram of the homemade scratch apparatus.

The samples for friction and wear tests were fabricated by placing epoxy composites to a cylindrical silicone mold with a diameter of $30 \mathrm{~mm}$. After $24 \mathrm{~h}$ for curing, the samples were removed from the mold. The test surfaces of all samples were treated with 2000 mesh sandpaper then cleaned with ethanol prior to the friction test.

\subsection{The Characterization of Microcapsules}

The FTIR spectra of the microcapsules, LO, polyurethane resin, and the extracted core were recorded by using FTIR spectrometer (Frontier, Perkin Elmer, Shanghai, China) in attenuated total 
reflectance (ATR) mode with the range of 4000 to $650 \mathrm{~cm}^{-1}$. The core material was separated from broken microcapsules by centrifugation with EtOAc.

The TG curves of microcapsules, LO and polyurethane resin were recorded by using thermogravimetric analyzer (DTG-60(H), SHIMADZU, Kyoto, Japan) in $\mathrm{N}_{2}$ environment. The sample mass was approximately $5 \mathrm{mg}$ and the test temperature range was 40 to $600{ }^{\circ} \mathrm{C}$ with a heating rate of $10{ }^{\circ} \mathrm{C} / \mathrm{min}$.

The morphology of microcapsules was observed by a scanning electron microscope (SEM, SU8020, Hitachi). Microcapsules were spread on conductive tape and sputtered with gold. To observe the internal structure, some of the microcapsules were intentionally broken.

The size distribution of microcapsules was measured by a Mastersizer particle size analyzer (Mastersizer 2000, Malvern).

\subsection{The Characterization of Coatings with Microcapsules}

The corrosive protection of the coating to metal substrates after being scratched was evaluated by neutral salt spray test. Prior to the test, the fringe and back of all samples were covered with paraffin wax. The samples were exposed to $5 \mathrm{wt} . \%$ of $\mathrm{NaCl}$ fog at $35 \pm 1{ }^{\circ} \mathrm{C}$ for $1,2,3$, and 4 weeks according to ISO 9227-2017.

The corrosion resistance behavior of the scratched coating in $3.5 \mathrm{wt} . \% \mathrm{NaCl}$ solution was evaluated by EIS. EIS measurement was performed on the electrochemistry workstation (CHI750E, Shanghai, China) via a three-electrode system. The reference electrode, counter electrode, and working electrode were assumed by saturated calomel electrode $\left(\mathrm{Hg} / \mathrm{Hg}_{2} \mathrm{Cl}_{2} / \mathrm{KCl}\right)$, platinum foil, and the sample with an exposed district of $9.07 \mathrm{~cm}^{2}$, respectively. A frequency range of $10^{-2}$ to $10^{5} \mathrm{~Hz}$ and an AC amplitude of $10 \mathrm{mV}$ were selected. The software Zview was carried to fit the EIS results.

The self-lubricating ability of the coating was evaluated by a linear reciprocating ball-on-disk friction wear tests performed on a tribometer (UMT-TriboLab, BRUKER, Milan, Italy) at ambient temperature. The samples were used to test disks and the GCr15 steel ball ( $\Phi 4 \mathrm{~mm})$ was taken as the counterpart. The test parameters are shown below: Applied load: $3 \mathrm{~N}$; stroke: $5 \mathrm{~mm}$; sliding frequency: $5 \mathrm{~Hz}$; velocity: $5 \mathrm{~cm} / \mathrm{s}$; test time: $30 \mathrm{~min}$. The friction coefficient of all samples was measured for analysis. The wear tracks were obtained through Nexview optical profilometer (ZYGO). The morphology of wear tracks was imaged through SEM.

\section{Results and Discussion}

\subsection{Synthesis and Morphology of Microcapsules}

Polyurethane microcapsules containing LO were synthesized via interfacial polymerization reaction occurred in an oil in water emulsion. L-75 is a TDI-based polyisocyanate that has previously been reported to encapsulate IPDI [35]. Trifunctional TMP dissolved in the aqueous phase as a cross-linking agent was favorable for higher cross-link density in the polymer structure. The $\mathrm{OH}$ of TMP and the NCO of L75 polymerize at the surfaces of microdroplets to form polyurethane, thereby encapsulating LO. GA acts as an emulsifier to allow the PU to deposit slowly on the surface of the microdroplets $[35,36]$. Feasible reaction mechanism for shell formation of polyurethane microcapsules is shown in Scheme 1. Figure 2 illustrates a schematic for the fabrication of polyurethane microcapsules containing LO via interfacial polymerization. 


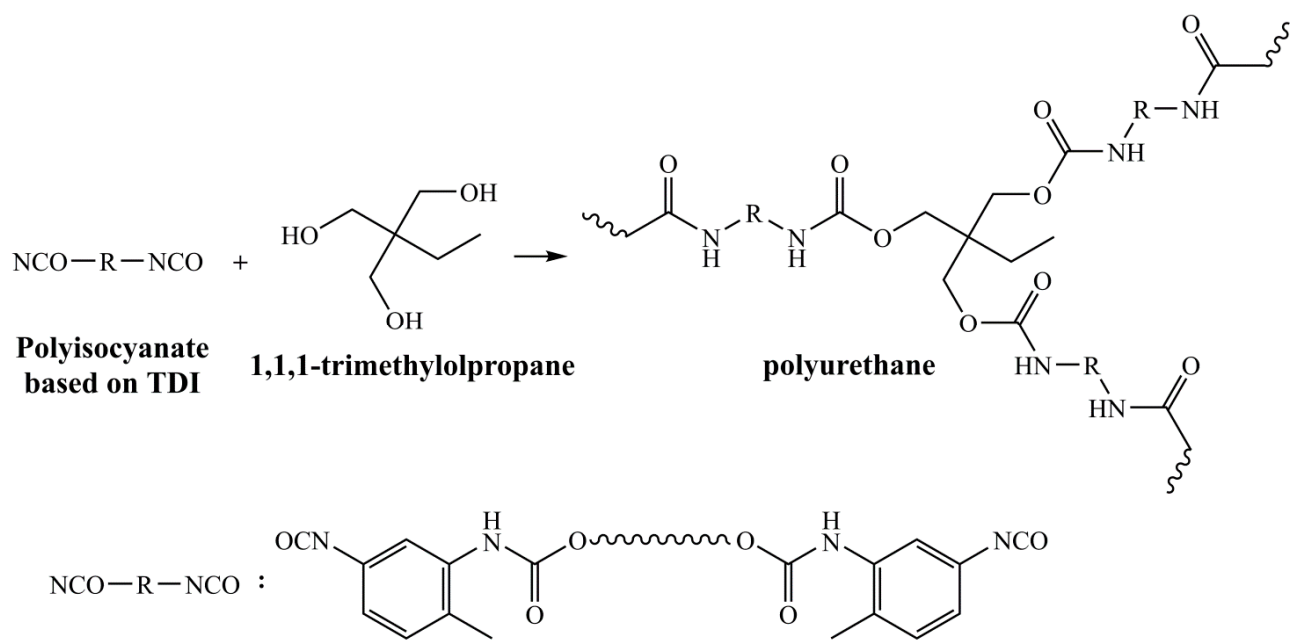

Scheme 1. Reaction scheme for shell formation of polyurethane microcapsules.

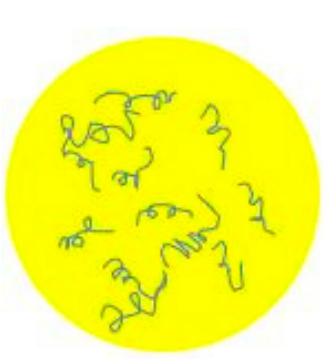

Linseed oil

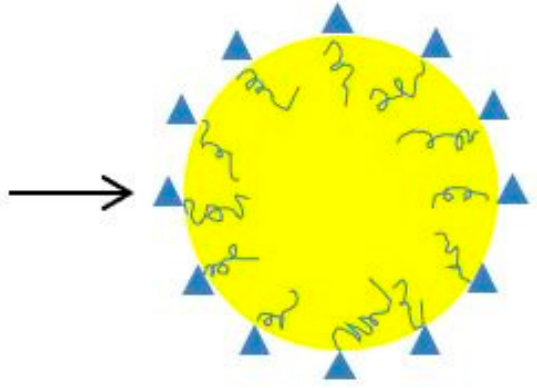

2. 3 Polyisocyanate

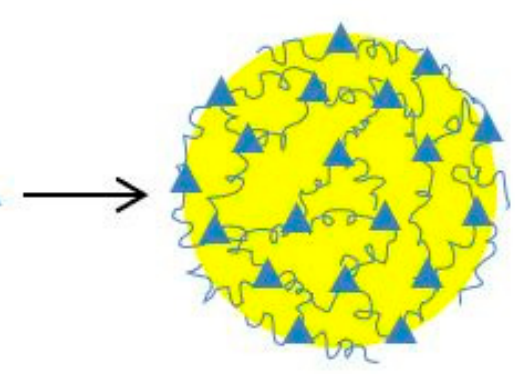

Trimethylolpropane

Figure 2. Schematic illustration of the microcapsules formation.

Microcapsules morphologies and particle size distribution were presented in Figure 3. It observes that the microcapsules present regular spherical shapes. Moreover, there is no adhesion between microcapsules, which is advantageous for their dispersion in the resin. Microcapsules show a smooth and compact surface, without holes and pits, indicating that the strength of microcapsules is sufficient to withstand the inevitable mechanical collision in preparation and use. It also provides a barrier to prevent LO from reacting with oxygen. A distinct hollow internal structure can be observed after microcapsules were crushed. The average diameter of microcapsules is $64.9 \mu \mathrm{m}$ (Figure $3 \mathrm{~d}$ ).

\subsection{FTIR Spectroscopy of Microcapsules}

The composition of the prepared microcapsules was analyzed by FTIR analysis. Figure 4 shows the FTIR spectrums of PU resin, LO, microcapsules, and the extracted core, respectively. The absorption bands of urethane groups (NHCOO) are exhibited in the spectrum: $\mathrm{N}-\mathrm{H}$ stretching appears at $3314 \mathrm{~cm}^{-1}, \mathrm{C}=\mathrm{O}$ stretching at $1715 \mathrm{~cm}^{-1}, \mathrm{~N}-\mathrm{H}$ bending at $1534 \mathrm{~cm}^{-1}, \mathrm{C}-\mathrm{N}$ stretching at $1224 \mathrm{~cm}^{-1}$, and $\mathrm{C}-\mathrm{O}$ stretching at $1070 \mathrm{~cm}^{-1}$ [35]. The stretching vibration of the benzene ring is clearly observed at $1600 \mathrm{~cm}^{-1}$, which confirmed the synthesis of PU resin. The spectrum of LO displayed the absorption bands of unsaturated C-H stretching at $3012 \mathrm{~cm}^{-1}$, saturated $\mathrm{C}-\mathrm{H}$ stretching at $2926 \mathrm{~cm}^{-1}$, saturated $\mathrm{C}-\mathrm{H}$ stretching at $2855 \mathrm{~cm}^{-1}, \mathrm{C}=\mathrm{O}$ stretching at $1745 \mathrm{~cm}^{-1}, \mathrm{C}-\mathrm{H}$ bending at $1457 \mathrm{~cm}^{-1}$, and C-COO stretching at $1162 \mathrm{~cm}^{-1}$ [27]. The absorption bands characteristics of PU resin and LO are conjointly presented in the spectrum of microcapsules. In addition, the core material was separated from broken microcapsules to further confirm the encapsulation of LO, and its FTIR spectrums as showed in Figure $4 \mathrm{~b}$. The spectrum of the LO matched the extracted core at the characteristic peak, confirming that LO was successfully encapsulated by PU shell. 

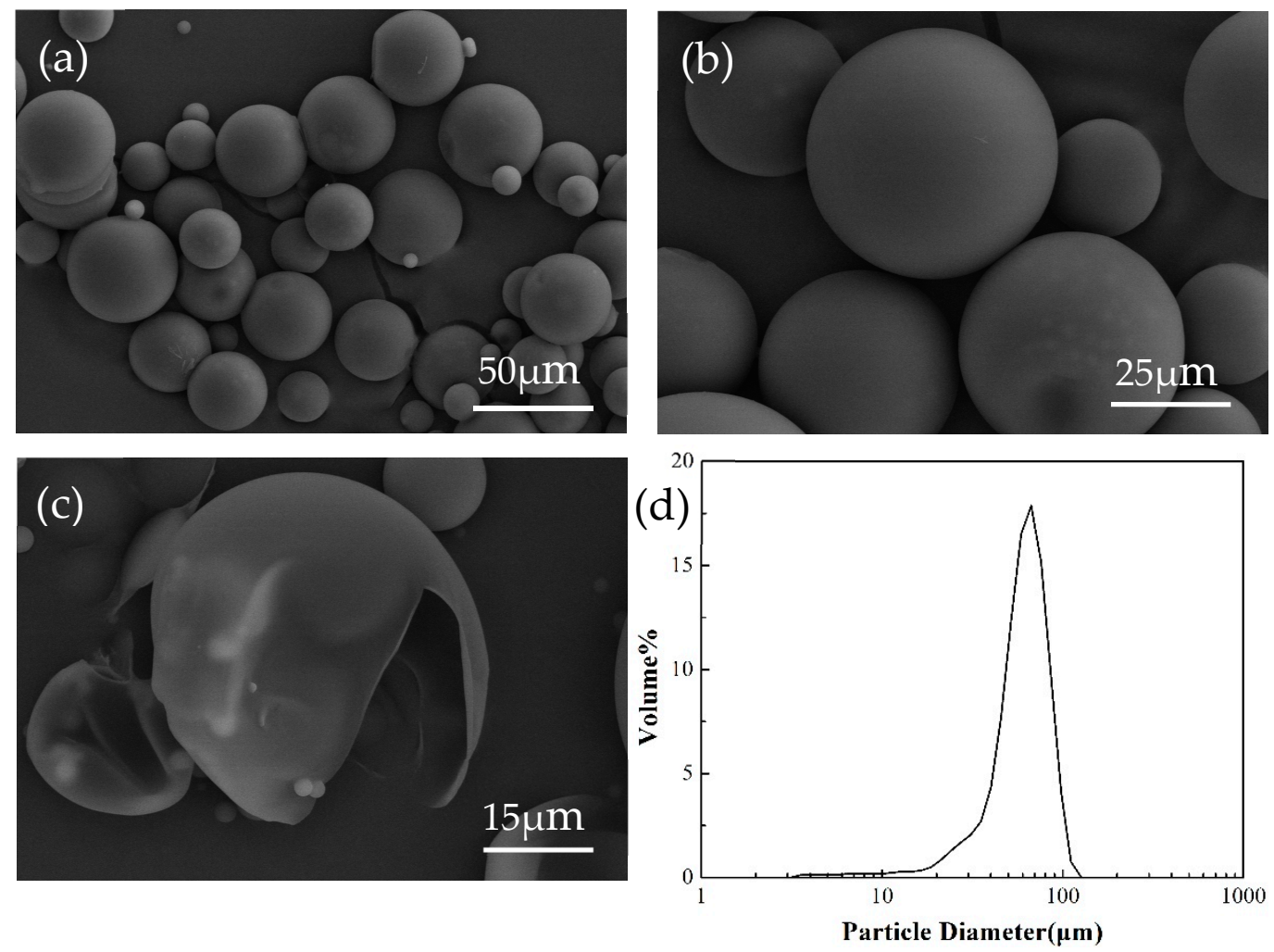

Figure 3. The morphology of linseed oil (LO) microcapsules: (a) overview of microcapsules with distributed diameters, (b) microcapsules magnification, (c) hollow structure of microcapsules, and (d) distribution of diameter of microcapsules.
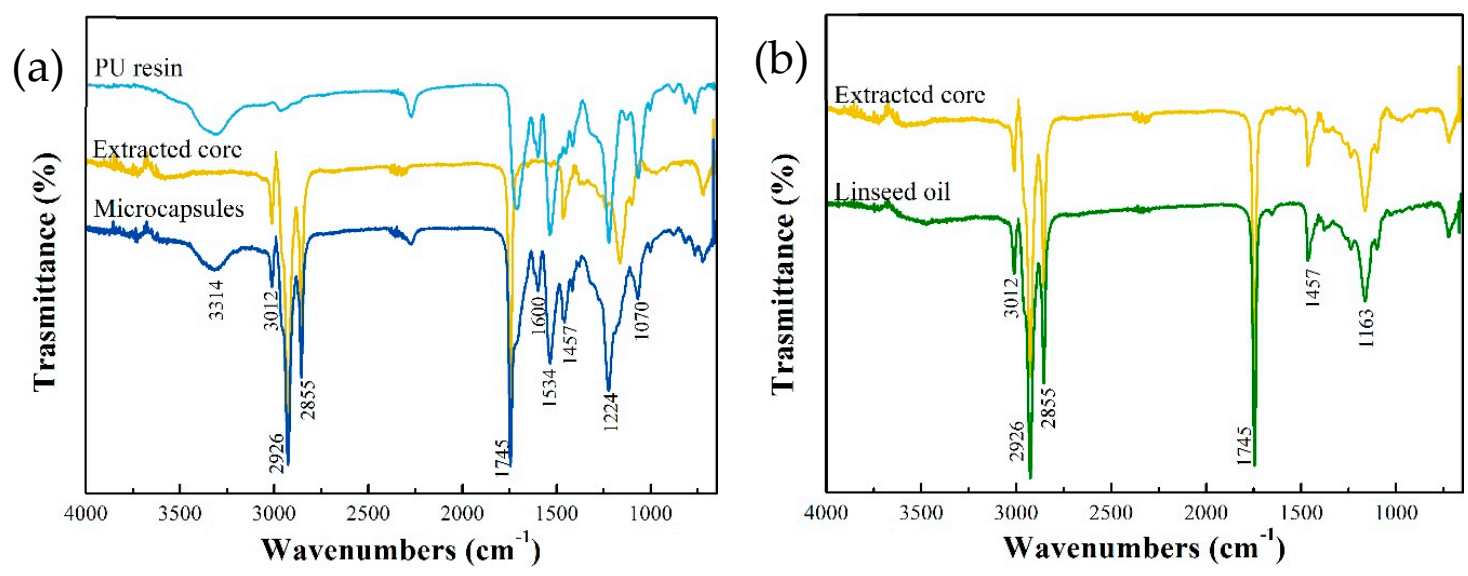

Figure 4. (a) FTIR spectra of Polyurethane (PU) resin, LO, and microcapsules containing core material.

(b) FTIR spectra of extracted core and LO.

\subsection{Thermal Stability of Microcapsules}

To determine the service temperature range and core content of microcapsules, its thermal properties were investigated by TGA. The TG curves of PU resin, LO, and microcapsules are exhibited in Figure 5a. The first derivative of TG curves to temperature is showed in Figure 5b. LO underwent decomposition in the range of 300 to $480^{\circ} \mathrm{C}$, and its maximum decomposition rate appears at $426{ }^{\circ} \mathrm{C}$, indicating better thermal stability compare to frequently used core materials such as epoxy [14], HMDI [18], and IPDI [22]. The PU shell is initially degraded at $250{ }^{\circ} \mathrm{C}$ and ended at $470{ }^{\circ} \mathrm{C}$, achieving a maximum decomposition rate at $306^{\circ} \mathrm{C}$. It can be concluded that the weight loss trend of the microcapsules is consistent with that of PU below $300{ }^{\circ} \mathrm{C}$. With the temperature rising to $300{ }^{\circ} \mathrm{C}$, 
a significant decrease in the mass of microcapsules was observed for the joint decomposition of the PU shell and the LO. Thermal stability of microcapsules is between the PU shell and LO. Accordingly, we can conclude that the microcapsules encapsulating LO was successfully fabricated. And PU microcapsules are more thermally stable than UF microcapsules, which experience a weight loss at $\sim 100-200{ }^{\circ} \mathrm{C}$ due to the release of free formaldehyde and formaldehyde split from ether groups [37-40].
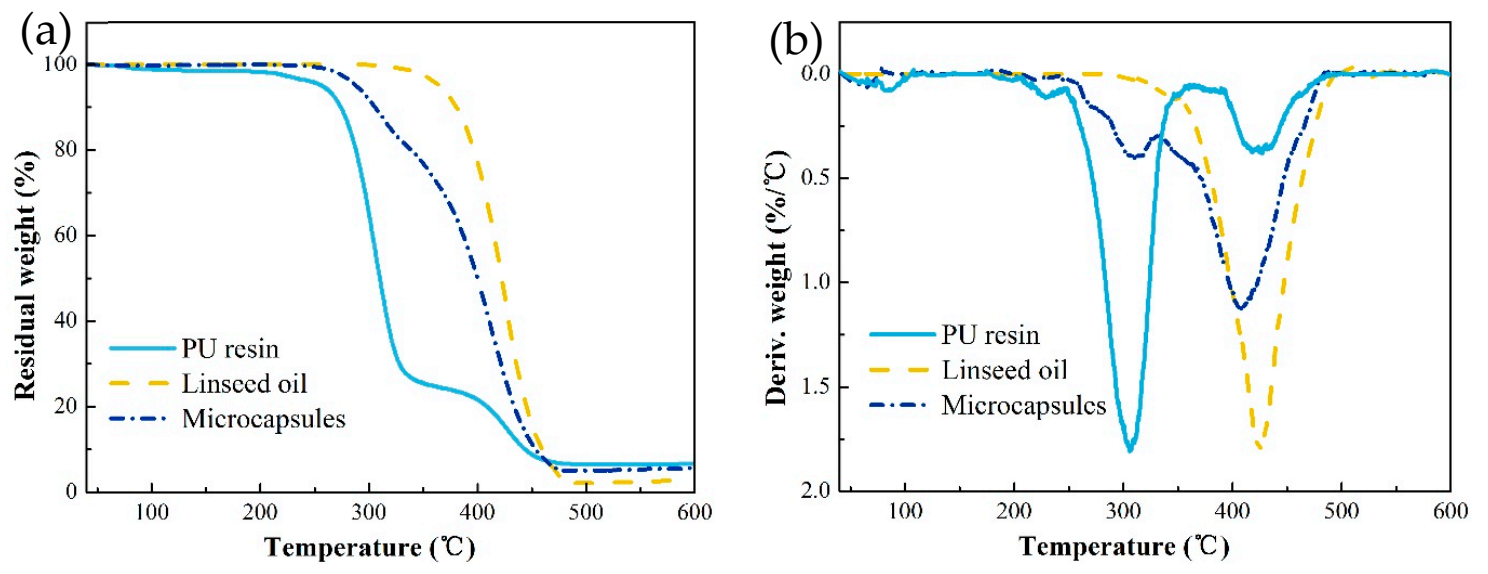

Figure 5. Thermal gravimetric (TG) (a) and differential thermogravimetric (DTG) (b) of LO, PU resin, and microcapsules containing core material.

The core content of microcapsules is calculated by the TG curves. There is nearly no mass loss of $\mathrm{LO}$ at $300{ }^{\circ} \mathrm{C}$, and the mass loss of microcapsules at the corresponding temperature can be attributed entirely to the decomposition of PU shell. Therefore, the ratio of the mass loss of microcapsules to PU shell equals to the shell content, and the core content is correspondingly obtained. The core content of microcapsules is calculated as high as $74 \mathrm{wt} . \%$, indicating that the encapsulation is effective.

\subsection{Salt Spray Test}

The systematic neutral salt spray tests were conducted to evaluate the self-healing property of the coating containing LO-loaded microcapsules. The pure epoxy coating and the coatings with microcapsules were exposed to salt spray for $0,1,2,3$, and 4 weeks, and the results are shown in Figure 6 . The rust phenomenon became more serious as the exposure time increases, and the corrosion resistance of coatings improved as the increase of microcapsules content. After 1 week exposure, obvious rust marks appeared in the scratched areas of the pure epoxy sample. In comparison, coatings with microcapsules showed slight rust. After 4 weeks, the scratched areas of the pure epoxy sample have been completely covered by corrosion products and obvious rust blisters showed around it. The coatings with microcapsules exhibit varying degrees of corrosion that is far less visible than the pure epoxy. Among them, the coating containing $20 \mathrm{wt} . \%$ microcapsules displayed the best corrosion protection to steel substrate, which showed no evident rust 4 weeks after explosion. Therefore, the coating containing microcapsules have self-healing performance and it is positively correlated with microcapsule content. This may be due to the higher the content of microcapsules, the more LO is used to fill the scratched areas and provide a denser barrier.

To further confirm the self-healing of coating containing microcapsules, the scratched area in pure epoxy coating and the coating with $10 \mathrm{wt} . \%$ microcapsules were observed by SEM. Artificial scratch of the coating with $10 \mathrm{wt} . \%$ microcapsules is covered by a new film, whereas that of the pure epoxy coating is not observed, as shown in Figure 7. After the coating containing microcapsules is damaged, the encapsulated $\mathrm{LO}$ is released to the microcracks by capillary action and polymerized with oxygen to heal the crack. The scheme of the self-healing procedure is indicated in Figure 7a-c. It provides a new barrier, while the pure epoxy keeps the scratched portion bare. Obviously, the leakproofness of this new barrier is greatly affected by the microcapsules content. 


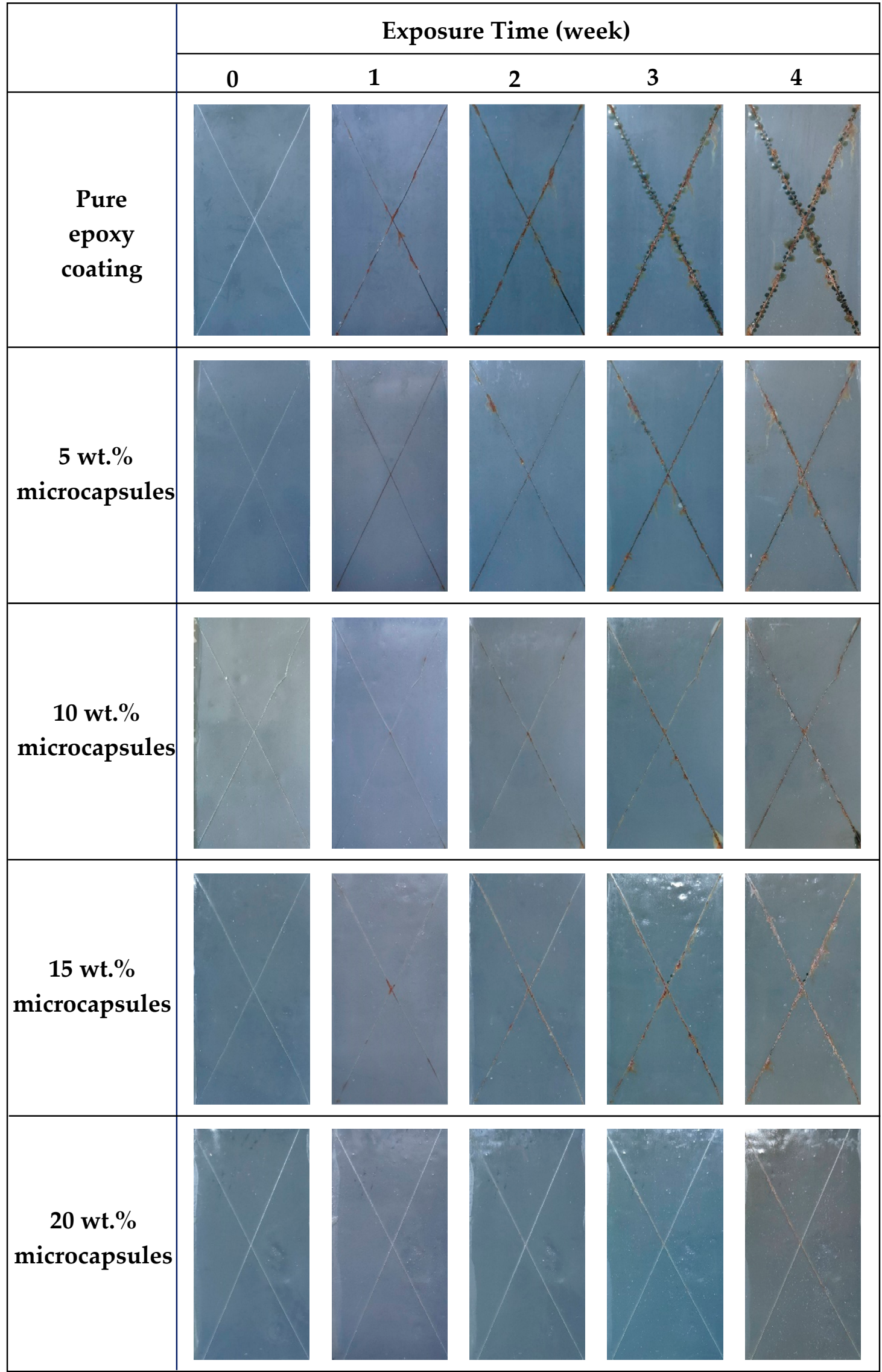

Figure 6. Salt spray test results for pure epoxy coatings and self-healing coatings containing 5, 10, 15, and $20 \mathrm{wt} . \%$ microcapsules after $0,1,2,3$, and 4 weeks exposure. 

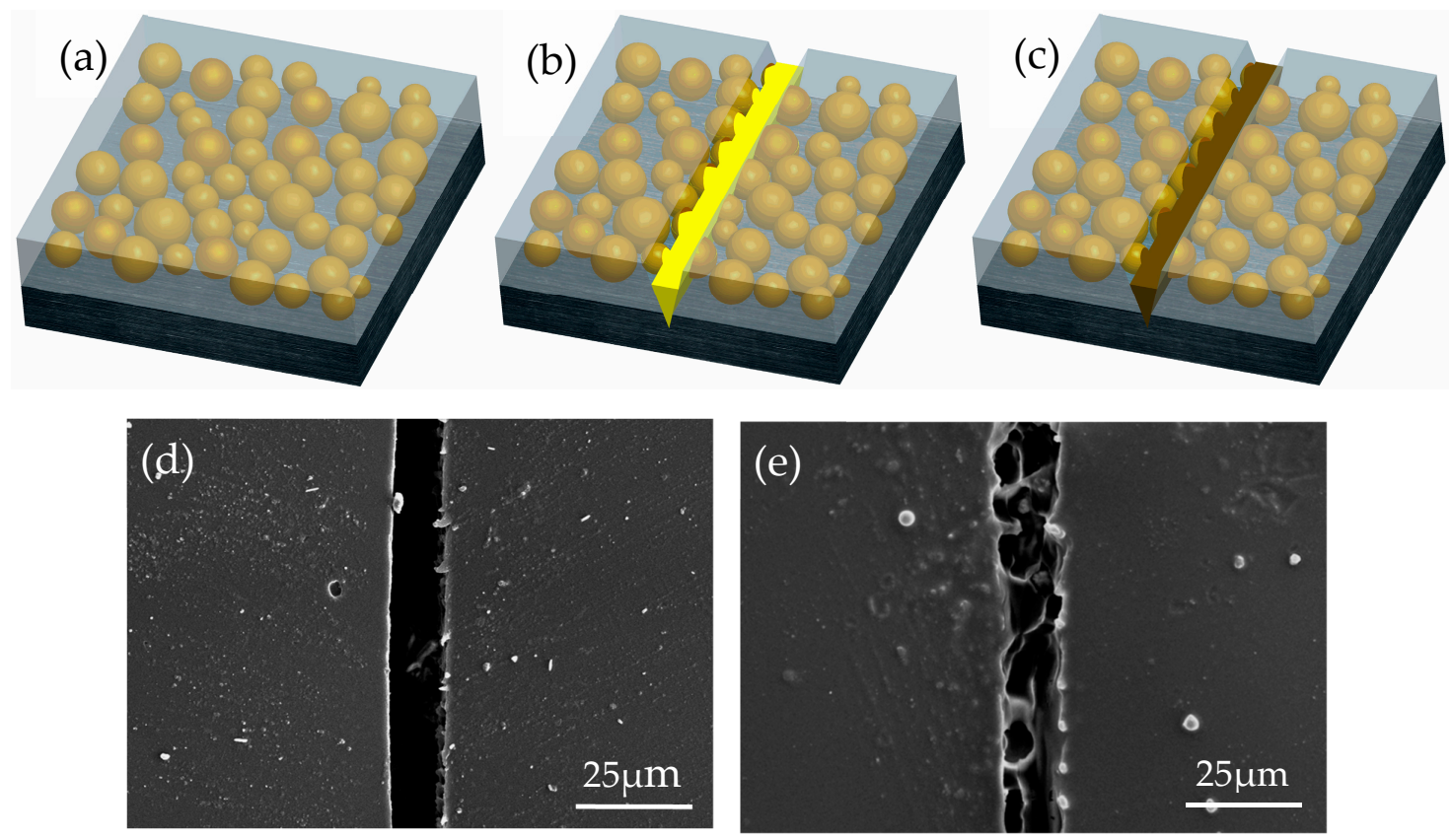

Figure 7. Scheme of the self-healing process. (a) Self-healing coating containing microcapsules on a metallic substrate. (b) Damage of the coating; release of the encapsulated LO on the metal surface. (c) A crack healed by the polymerization of LO with oxygen. SEM micrographs of the scratched area of the (d) pure epoxy coating and (e) self-healing coating with $10 \mathrm{wt} \%$ microcapsules.

\subsection{EIS Measurement}

The self-healing of coatings with 5, 10, 15, and $20 \mathrm{wt} . \%$ microcapsules was further evaluated with EIS technique. The Nyquist and Bode diagrams are displayed in Figure 8. The equivalent circuit is equipped with the constant phase element $Q(\mathrm{CPE})$, which is calculated by the following formula [41],

$$
Z_{Q}=T^{-1}(j \omega)^{-P},
$$

where $T$ is a constant with dimension $\mathrm{F} \cdot \mathrm{cm}^{-2} \mathrm{~s}^{\mathrm{P}-1}, j=(-1)^{1 / 2}, \omega$ is the angular frequency, and $P$ is the exponent related to the degree of frequency dispersion.

The equivalent circuit models were shown in Figure 9. The circuit model parameters were presented in Table 1. $R_{s}$ is the solution resistance. $Q_{d l}$ and $R_{c t}$ are the electric double-layer capacitor and charge-transfer resistance. $Q_{\text {healing }}$ and $R_{\text {healing }}$ represent the healing resistance and healing capacitor. $W$ is the Warburg impedance.

After immersion for 0 days, a single capacitive loop of pure epoxy was observed in the corresponding Nyquist plot. The electrochemical response spectra of pure epoxy sample shows the one time constant, and it was fitted with model 1. In comparison, the EIS results of coatings with 5, 10,15 , and $20 \mathrm{wt} . \%$ microcapsules were fitted with model 2 , taking into account the healing capacitance and healing resistance. Their radius of single capacitive loop $\left(R_{\text {healing }}\right)$ were above $10^{10} \Omega \cdot \mathrm{cm}^{2}$, much higher than the pure epoxy $\left(R_{c t}=3.62 \times 10^{6} \Omega \cdot \mathrm{cm}^{2}\right)$. Correspondingly, the Bode plots show higher impedance modulus $(|Z|)$ in the $10^{-2}$ to $10^{5} \mathrm{~Hz}$ range for the coatings with microcapsules than the pure epoxy coating. Moreover, the phase angle of the coatings with microcapsules is close to $-90^{\circ}$ over a wide range, which means that the coatings with microcapsules have good protection performance after being scratched. 

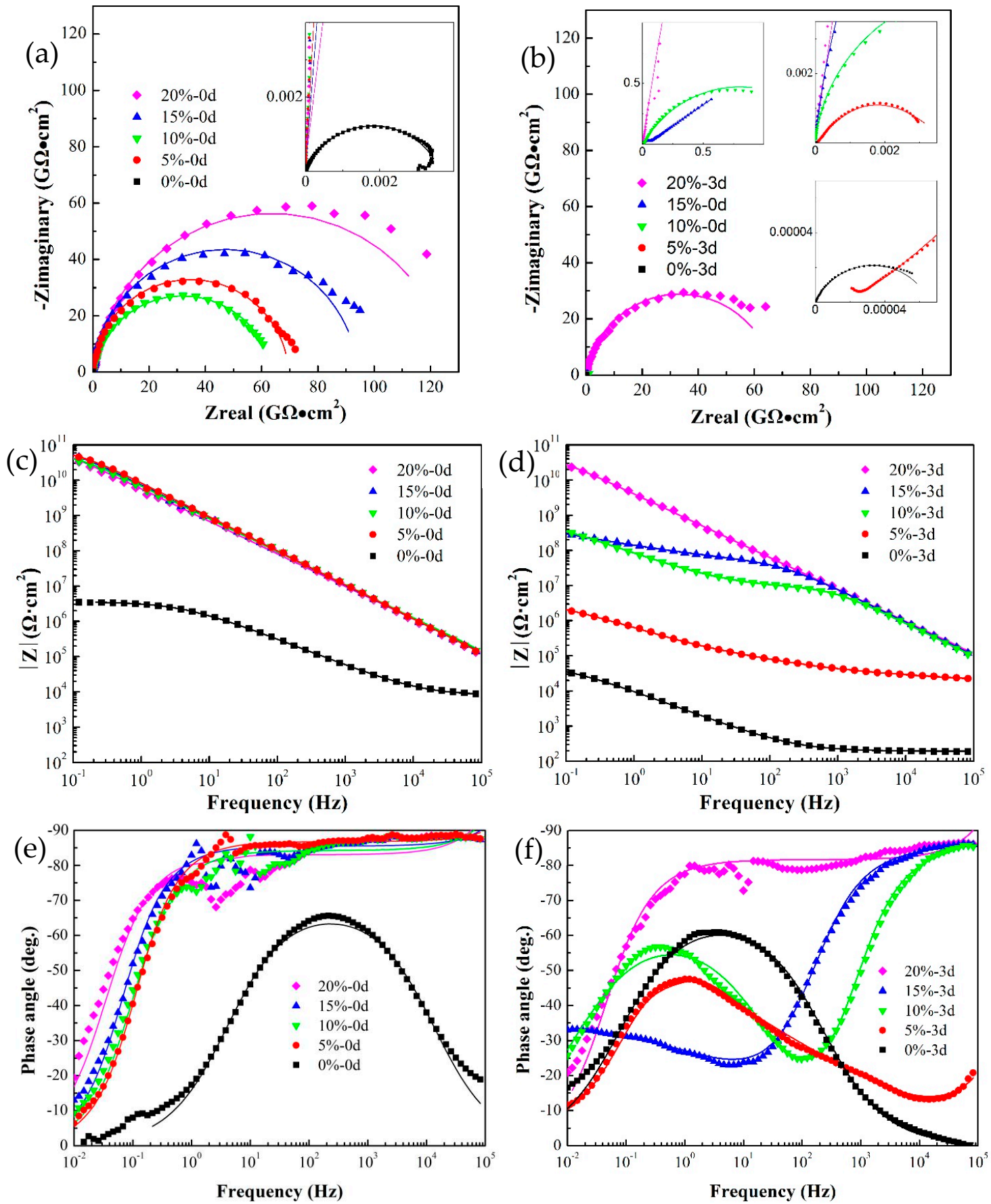

Figure 8. Nyquist plots $(\mathbf{a}, \mathbf{b})$, Bode impedance $(\mathbf{c}, \mathbf{d})$, and Bode phase $(\mathbf{e}, \mathbf{f})$ of pure epoxy coatings and self-healing coatings containing 5, 10, 15, and $20 \mathrm{wt}$.\% microcapsules after scratching and immersing in $3.5 \% \mathrm{NaCl}$ electrolyte for $0(\mathrm{a}, \mathrm{c}, \mathrm{e})$ and $3(\mathrm{~b}, \mathrm{~d}, \mathrm{f})$ days. The points represent experimental data and the continuous lines with the same color represent the fits. 
After immersion for 3 days, the pure epoxy sample shows a decrease in the value of $|Z|_{f=0.01 \mathrm{~Hz}}$ to $10^{4} \Omega \cdot \mathrm{cm}^{2}$. Besides, the Bode phase plots show the single peak moving to a lower frequency. It indicated that the pure epoxy sample takes a further reduction in corrosion resistance. The $|Z|_{f=0.01 \mathrm{~Hz}}$ of the coating with $5 \mathrm{wt} . \%$ microcapsules dropped sharply to $10^{6} \Omega \cdot \mathrm{cm}^{2}$. Furthermore, the Warburg impedance characteristics are presented in the intermediate frequency, and the EIS result was fitted with model 4 . This indicated that the coating has continuous diffusion channels for corrosive medium, and its corrosion protection performance is basically ineffective but still better than the pure epoxy sample. The electrochemical response of the coating with 10 and $15 \mathrm{wt} . \%$ microcapsules transitioned to double capacitive loop, illustrating that the electrolyte solution has penetrated into the metal interface through the weak point of the healing layer and the corrosion reaction has begun. The EIS results of them were fitted by Model 3. However, the coating with 15wt.\% microcapsules has better corrosion resistance, since the capacitive loop at high frequency shows a larger diameter and the capacitive loop in low frequency is not fully formed. Model 2 is still suitable for the EIS results of the coating with $20 \mathrm{wt} . \%$ microcapsules, $R_{\text {healing }}$ only dropped from $10^{11}$ to $10^{10} \Omega \cdot \mathrm{cm}^{2}$, maintaining good corrosion resistance. In conclusion, he coating with microcapsules exhibited self-healing ability, which was positively correlated with the microcapsules content. This is in agreement with the salt spray test results.
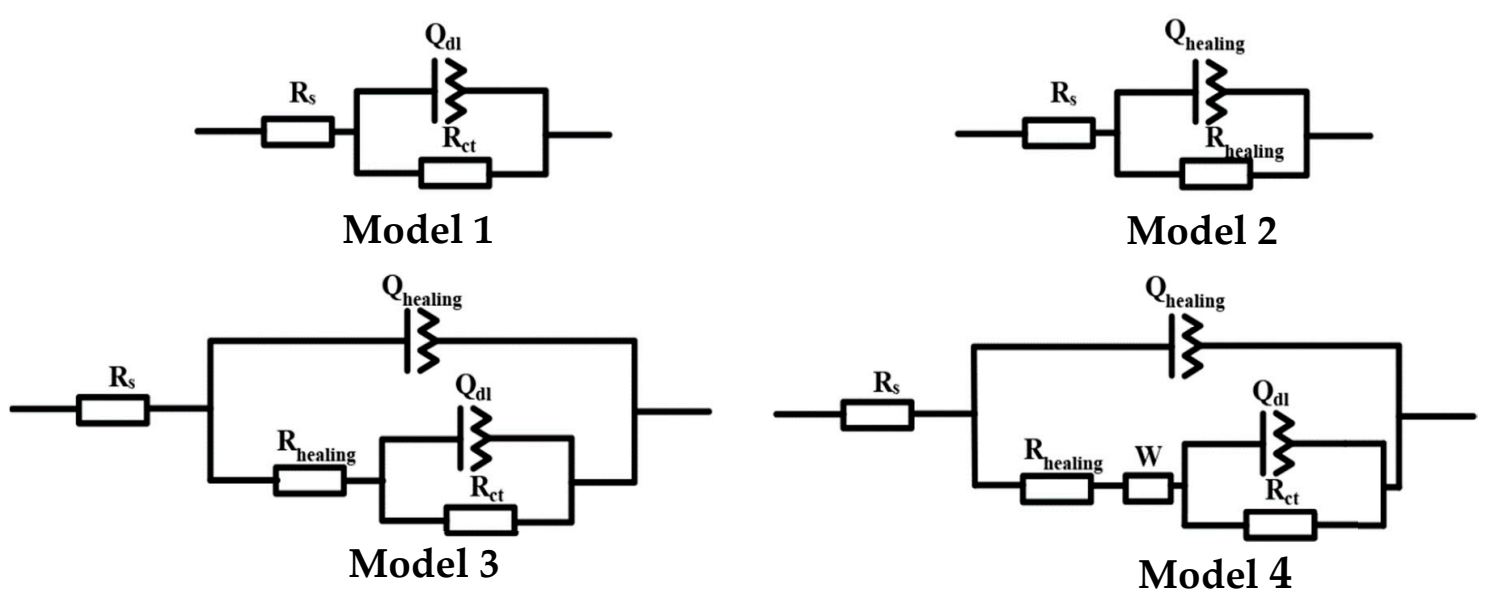

Figure 9. Four models of equivalent circuits.

Table 1. Circuit model parameters for the scratched coatings with 5, 10, 15, and $20 \mathrm{wt} . \%$ microcapsules at 0 and 3 days exposure time to $3.5 \% \mathrm{NaCl}$ solution.

\begin{tabular}{|c|c|c|c|c|c|c|c|c|}
\hline \multirow[b]{2}{*}{ Time } & \multirow{2}{*}{$\begin{array}{l}\text { Microcapsules } \\
\text { Content (wt.\%) }\end{array}$} & \multirow{2}{*}{$\begin{array}{l}R_{\text {healing }} \\
\left(\Omega \bullet \mathrm{cm}^{2}\right)\end{array}$} & \multirow[b]{2}{*}{$R_{c t}\left(\Omega \bullet \mathrm{cm}^{2}\right)$} & \multicolumn{2}{|c|}{$Q_{\text {healing }}$} & \multicolumn{2}{|l|}{$Q_{d l}$} & \multirow{2}{*}{$\frac{W}{R\left(\Omega \bullet \mathrm{cm}^{2}\right)}$} \\
\hline & & & & $\begin{array}{c}T \\
\left(\mathrm{~F} \bullet \mathrm{cm}^{-2} \mathrm{~s}^{\mathrm{P}-1}\right)\end{array}$ & $P$ & $\begin{array}{c}T \\
\left(\mathrm{~F} \bullet \mathrm{cm}^{-2} \mathrm{~s}^{\mathrm{P}-1}\right)\end{array}$ & $P$ & \\
\hline \multirow{5}{*}{0 day } & 0 & - & $3.62 \times 10^{6}$ & - & - & $2.39 \times 10^{-8}$ & 0.756 & - \\
\hline & 5 & $6.96 \times 10^{10}$ & - & $2.01 \times 10^{-11}$ & 0.964 & - & - & - \\
\hline & 10 & $6.38 \times 10^{10}$ & - & $2.90 \times 10^{-11}$ & 0.896 & - & - & - \\
\hline & 15 & $9.43 \times 10^{10}$ & - & $2.36 \times 10^{-11}$ & 0.950 & - & - & - \\
\hline & 20 & $1.27 \times 10^{11}$ & - & $3.20 \times 10^{-11}$ & 0.922 & - & - & - \\
\hline \multirow{5}{*}{3 days } & 0 & - & $6.59 \times 10^{4}$ & - & - & $2.57 \times 10^{-5}$ & 0.729 & - \\
\hline & 5 & 17740 & $3.18 \times 10^{6}$ & $1.83 \times 10^{-7}$ & 0.358 & $2.99 \times 10^{-8}$ & 0.031 & $8.17 \times 10^{5}$ \\
\hline & 10 & $9.42 \times 10^{6}$ & $1.58 \times 10^{9}$ & $3.74 \times 10^{-11}$ & 0.937 & $3.44 \times 10^{-9}$ & 0.683 & - \\
\hline & 15 & $4.03 \times 10^{7}$ & $1.35 \times 10^{11}$ & $3.33 \times 10^{-11}$ & 0.940 & $4.53 \times 10^{-9}$ & 0.392 & - \\
\hline & 20 & $6.69 \times 10^{10}$ & - & $4.64 \times 10^{-11}$ & 0.907 & - & - & - \\
\hline
\end{tabular}




\subsection{Self-Lubricating Mechanism}

The self-lubricity of the coating with different concentrations of microcapsules was evaluated by a friction wear test. Figure 10 shows the evolution of friction coefficients with sliding times for the pure epoxy coating and coatings with different concentrations of microcapsules, under dry sliding against GCr15 steel balls. The corresponding three-dimensional white light interferogram and scanning electron micrograph are shown in Figures 11 and 12.

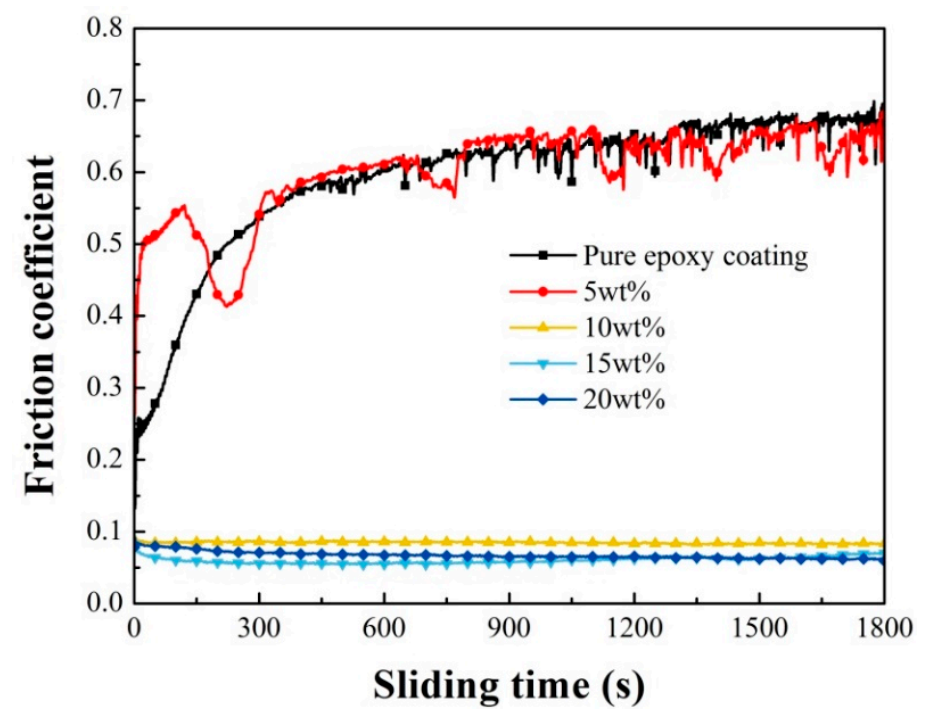

Figure 10. Friction coefficients of pure epoxy coating and the coatings with 5, 10, 15, and 20 wt.\% microcapsules as a function of sliding time.

The friction coefficient of pure epoxy coating increases during the initial period and then gradually stabilizes, around 0.673. During the running-in period, the surface microprotrusions are worn away, which increases the contact area and causes more serious interlock behavior, resulting in an augment in the friction coefficient at initial. After addition of 5\% microcapsules, the friction record is similar to pure epoxy coating, but exhibited apparent fluctuations. As the addition of microcapsules are 10, 15 , and $20 \mathrm{wt} . \%$, the friction coefficient of the coatings remained basically unchanged with sliding time, and is very low at $\sim 0.07$. The coating by incorporating $5,10,15$, and $20 \mathrm{wt} \%$ microcapsules affords $3.36 \%, 87.52 \%, 90.11 \%$, and $90.65 \%$ friction coefficient reduction, respectively. The addition of LO microcapsules (in $10 \mathrm{wt}$ \%) show stronger self-lubricating effects than HMDI (78.9\%) [18] and tung oil (17.3\%) [30]. Consequently, coatings exhibited excellent abrasion resistance as the content of microcapsules is increased to $10 \mathrm{wt}$ \% or more.

The linear wear tracks gradually become shallower follow by the increase of microcapsules content as shown in Figure 11. Wear scars are basically invisible when the microcapsule content reached $10 \mathrm{wt} . \%$ or more, which was consistent with the tendency of friction coefficient. The morphological observation of the worn surface of all samples by SEM revealed that pure epoxy coating and the coating with 5 wt.\% microcapsules exhibit fatigue wear characteristics. There are distinct grooves, which are filled with a large area of microcrack network, formed by the removal of materials along the sliding direction; the microcracks are generally perpendicular to the sliding direction. This is caused by repeated sliding of the GCr15 ball under load resulting in cyclic stress concentration on the surface of the coating. When the addition amount microcapsules less than $5 \mathrm{wt} \%$, the LO is not sufficient to increase the abrasion resistance of the coating. The coatings containing 10, 15, and $20 \mathrm{wt}$. \% shows the relatively smooth and flat wear surface without visible microcracks and wear debris, indicating only slight adhesive wear. The aforementioned phenomenon was mainly attributed to the LO releasing of microcapsules after being worn out during the wear process. The LO forms a transfer film between the sample and the friction pair due to physical adsorption, which separates the friction interfaces that 
contact each other and reduces the shear resistance. Figure 13 shows a schematic of the self-lubricating process. Therefore, the coatings with microcapsules exhibit self-lubricating properties and become superior with increasing content.
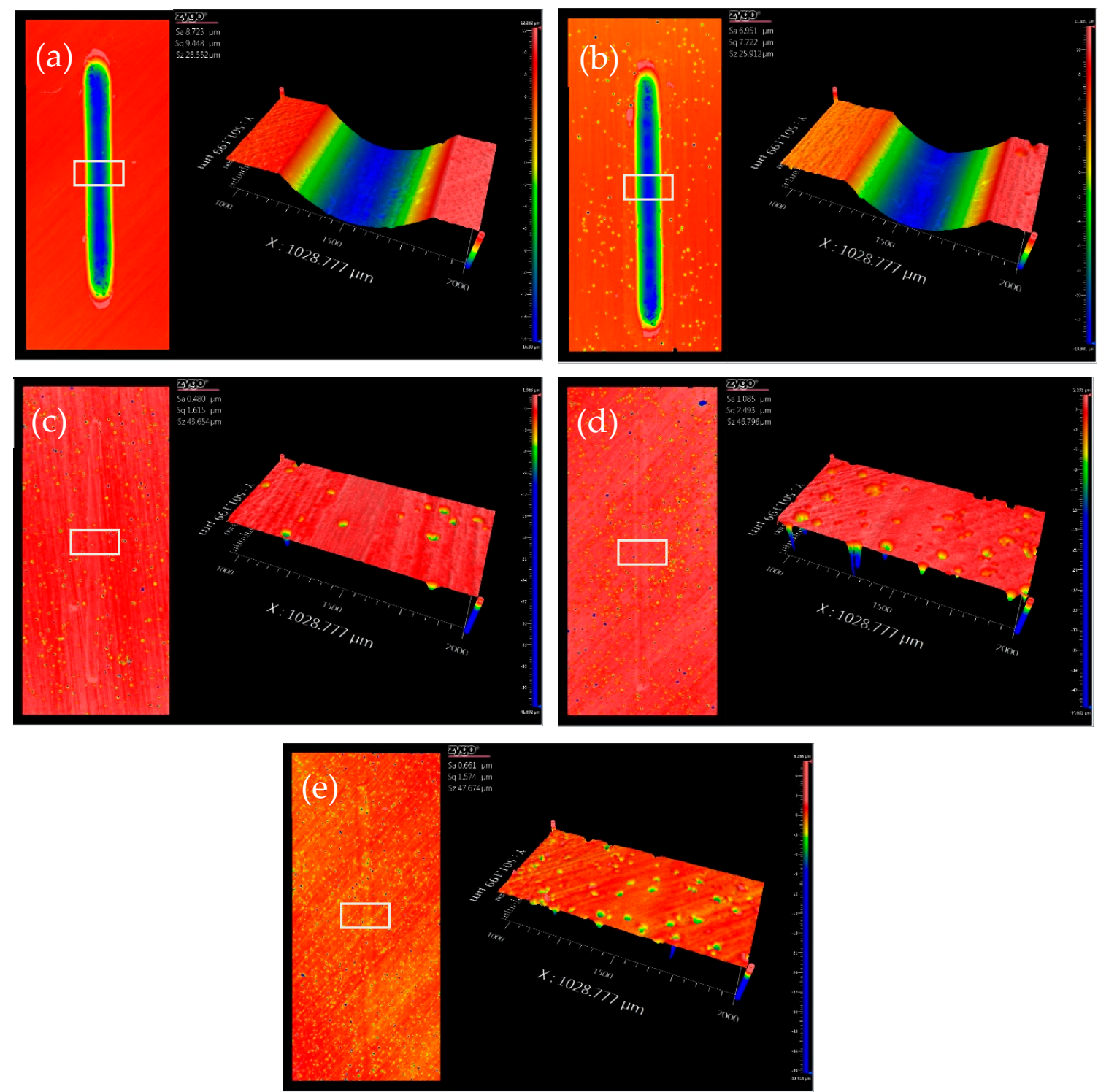

Figure 11. The topographies of samples after tribological tests containing (a) a pure epoxy coating and (b-e) a coating with 5, 10, 15, and $20 \mathrm{wt}$ \% microcapsules and the respective partial enlargement. 

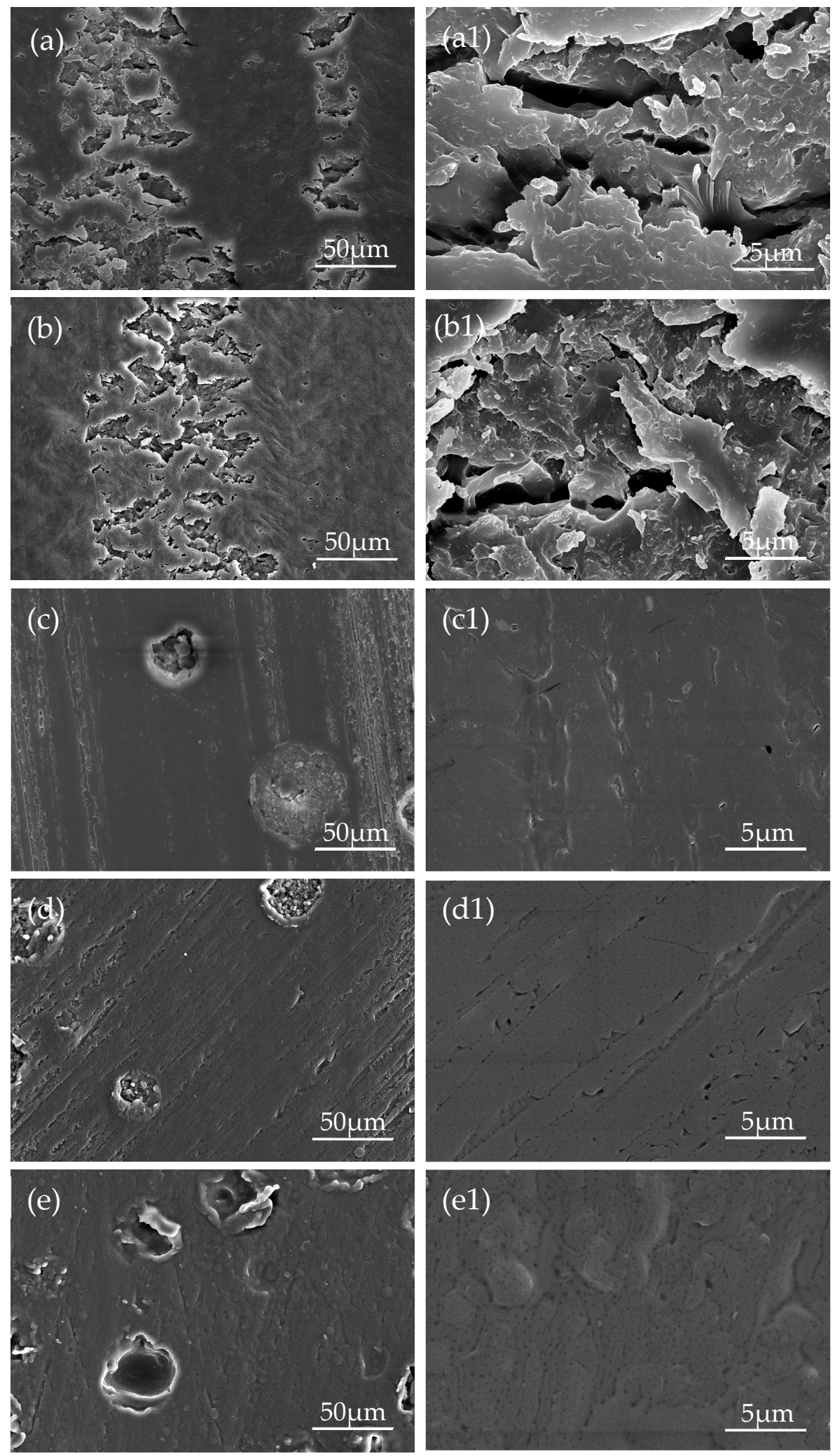

Figure 12. SEM micrographs showing worn surfaces of samples slid against a GCr15 steel ball of $4 \mathrm{~mm}$ in diameter in a linear path of $5 \mathrm{~mm}$ in length for $\sim 1800 \mathrm{~s}$ at a sliding speed of $5 \mathrm{~cm} / \mathrm{s}$, under a load of $3 \mathrm{~N}$ : (a) pure epoxy coating, (b-e) coating with 5, 10, 15 and $20 \mathrm{wt} . \%$ microcapsules, and the respective partial enlargement (a1, b1, c1, d1, and e1). 

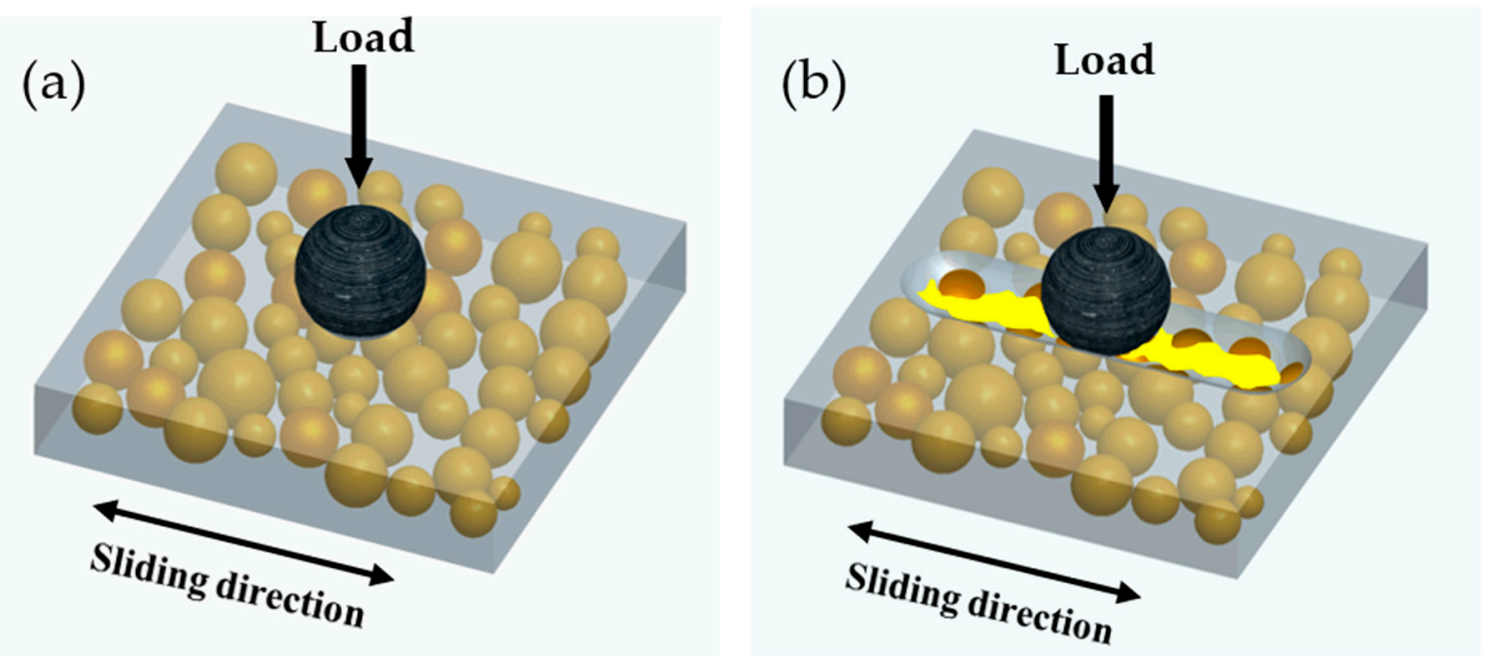

Figure 13. Schematic of the self-lubricating process: (a) Self-lubricating coating with microcapsules containing linseed oil slid against a steel ball under a load. (b) Damage of the coating; release of the encapsulated linseed oil at the friction interface.

\section{Conclusions}

In this study, the corrosion resistance and friction wear performances of epoxy composites with different contents of PU microcapsules containing bifunctional LO were systematically investigated. The coating is embedded with LO microcapsules exhibited excellent self-healing corrosion resistance in the EIS and salt spray tests; the corrosion resistance of the healing film formed within the scratched area was positively correlated with the microcapsules content. Furthermore, excellent self-lubricating properties of the epoxy resin with $10 \mathrm{wt} . \%$ microcapsules or more have also been confirmed. The epoxy coating shows $86.8 \%$ friction coefficient reduction after the concentration of microcapsules reached $10 \mathrm{wt} . \%$. These results indicate that the addition of PU microcapsules containing $\mathrm{LO}$ is an available approach to improve the corrosion resistance and the friction wear performances of the epoxy composites. It heralds the enormous potential of microcapsules encapsulating $\mathrm{LO}$ in the field of bifunctional coating for self-healing and self-lubrication.

Author Contributions: Conceptualization, W.L. and H.Y.; data curation, H.Y.; formal analysis, H.Y., Q.M., and F.G.; funding acquisition, W.L.; investigation, H.Y.; methodology, W.L., H.Y., Q.M., and F.G.; project administration, W.L.; resources, W.L.; supervision, W.L.; validation, H.Y., Q.M., and F.G.; visualization, H.Y.; writing一original draft, H.Y.; writing-review and editing, H.Y., Q.M., and W.L.

Funding: The research was financially supported by the Guangxi National Science Foundation of China (2016GXNSFDA380022), Major Science and Technology Projects in Guangxi (AA18118030, AA17204100), High Level Innovation Team and Outstanding Scholar Program in Guangxi Colleges (second batch), and the Project of Development of Science and Technology of Nanning (20181191-2).

Conflicts of Interest: The authors declare no conflicts of interest.

\section{References}

1. Wei, H.; Wang, Y.; Guo, J.; Shen, N.Z.; Jiang, D.; Zhang, X.; Yan, X.; Zhu, J.; Wang, Q.; Shao, L.; et al. Advanced micro/nanocapsules for self-healing smart anticorrosion coatings. J. Mater. Chem. A 2015, 3, 469-480. [CrossRef]

2. Ataei, S.; Khorasani, S.N.; Neisiany, R.E. Biofriendly vegetable oil healing agents used for developing self-healing coatings: A review. Prog. Org. Coat. 2019, 129, 77-95. [CrossRef]

3. Behzadnasab, M.; Mirabedini, S.M.; Esfandeh, M.; Farnood, R.R. Evaluation of corrosion performance of a self-healing epoxy-based coating containing linseed oil-filled microcapsules via electrochemical impedance spectroscopy. Prog. Org. Coat. 2017, 105, 212-224. [CrossRef] 
4. Zhang, F.; Ju, P.; Pan, M.; Zhang, D.; Huang, Y.; Li, G.; Li, X. Self-healing mechanisms in smart protective coatings: A review. Corros. Sci. 2018, 144, 74-88. [CrossRef]

5. Hasanzadeh, M.; Shahidi, M.; Kazemipour, M. Application of EIS and en techniques to investigate the self-healing ability of coatings based on microcapsules filled with linseed oil and $\mathrm{CeO}_{2}$ nanoparticles. Prog. Org. Coat. 2015, 80, 106-119. [CrossRef]

6. Suryanarayana, C.; Rao, K.C.; Kumar, D. Preparation and characterization of microcapsules containing linseed oil and its use in self-healing coatings. Prog. Org. Coat. 2008, 63, 72-78. [CrossRef]

7. Nazeer, A.A.; Madkour, M. Potential use of smart coatings for corrosion protection of metals and alloys: A review. J. Mol. Liq. 2018, 253, 11-22. [CrossRef]

8. Bekas, D.G.; Tsirka, K.; Baltzis, D.; Paipetis, A.S. Self-healing materials: A review of advances in materials, evaluation, characterization and monitoring techniques. Compos. Part B 2016, 87, 92-119. [CrossRef]

9. White, S.R.; Sottos, N.R.; Geubelle, P.H.; Moore, J.S.; Kessler, M.R.; Sriram, S.R.; Brown, E.N.; Viswanathan, S. Autonomic healing of polymer composites. Nature 2001, 409, 794. [CrossRef]

10. Samadzadeh, M.; Boura, S.H.; Peikari, M.; Kasiriha, S.M.; Ashrafi, A. A review on self-healing coatings based on micro/nanocapsules. Prog. Org. Coat. 2010, 68, 159-164. [CrossRef]

11. An, S.; Lee, M.W.; Yarin, A.L.; Yoon, S.S. A review on corrosion-protective extrinsic self-healing: Comparison of microcapsule-based systems and those based on core-shell vascular networks. Chem. Eng. J. 2018, 344, 206-220. [CrossRef]

12. Jin, H.; Mangun, C.L.; Griffin, A.S.; Moore, J.S.; Sottos, N.R.; White, S.R. Thermally stable autonomic healing in epoxy using a dual-microcapsule system. Adv. Mater. 2014, 26, 282-287. [CrossRef] [PubMed]

13. Caruso, M.M.; Blaiszik, B.J.; White, S.R.; Sottos, N.R.; Moore, J.S. Full recovery of fracture toughness using a nontoxic solvent-based self-healing system. Adv. Funct. Mater. 2010, 18, 1898-1904. [CrossRef]

14. Jin, H.; Mangun, C.L.; Stradley, D.S.; Moore, J.S.; Sottos, N.R.; White, S.R. Self-healing thermoset using encapsulated epoxy-amine healing chemistry. Polymer 2012, 53, 581-587. [CrossRef]

15. Mangun, C.L.; Mader, A.C.; Sottos, N.R.; White, S.R. Self-healing of a high temperature cured epoxy using poly(dimethylsiloxane) chemistry. Polymer 2010, 51, 4063-4068. [CrossRef]

16. Keller, M.W.; White, S.R.; Sottos, N.R. A self-healing poly(dimethyl siloxane) elastomer. Adv. Funct. Mater. 2007, 17, 2399-2404. [CrossRef]

17. Cho, S.H.; Andersson, H.M.; White, S.R.; Sottos, N.R.; Braun, P.V. Polydiniethylsiloxane-based self-healing materials. Adv. Mater. 2006, 18, 997-1000. [CrossRef]

18. Sun, D.; Chong, Y.B.; Chen, K.; Yang, J. Chemically and thermally stable isocyanate microcapsules having good self-healing and self-lubricating performances. Chem. Eng. J. 2018, 346, 289-297. [CrossRef]

19. Huang, M.; Yang, J. Facile microencapsulation of HDI for self-healing anticorrosion coatings. J. Mater. Chem. 2011, 21, 11123. [CrossRef]

20. Wang, W.; Xu, L.; Li, X.; Yang, Y.; An, E. Self-healing properties of protective coatings containing isophorone diisocyanate microcapsules on carbon steel surfaces. Corros. Sci. 2014, 80, 528-535. [CrossRef]

21. Yang, J.; Keller, M.W.; Moore, J.S.; White, S.R.; Sottos, N.R. Microencapsulation of isocyanates for self-healing polymers. Macromolecules 2010, 41, 9650-9655. [CrossRef]

22. Li, C.; Tan, J.; Gu, J.; Qiao, L.; Zhang, B.; Zhang, Q. Rapid and efficient synthesis of isocyanate microcapsules via thiol-ene photopolymerization in Pickering emulsion and its application in self-healing coating. Compos. Sci. Technol. 2016, 123, 250-258. [CrossRef]

23. Guo, M.; Li, W.; Han, N.; Wang, J.; Su, J.; Li, J.; Zhang, X. Novel dual-component microencapsulated hydrophobic amine and microencapsulated isocyanate used for self-healing anti-corrosion coating. Polymers 2018, 10, 319. [CrossRef] [PubMed]

24. Ma, Y.; Jiang, Y.; Tan, H.; Zhang, Y.; Gu, J. A rapid and efficient route to preparation of isocyanate microcapsules. Polymers 2017, 9, 274. [CrossRef] [PubMed]

25. Zheng, N.; Liu, J.; Li, W. TO/TMMP-TMTGE double-healing composite containing a transesterification reversible matrix and tung oil-loaded microcapsules for active self-healing. Polymers 2019, 11, 1127. [CrossRef] [PubMed]

26. Boura, S.H.; Peikari, M.; Ashrafi, A.; Samadzadeh, M.; Hatami Boura, S.; Peikari, M.; Ashrafi, A.; Samadzadeh, M.; Boura, S.H.; Peikari, M.; et al. Self-healing ability and adhesion strength of capsule embedded coatings-Micro and nano sized capsules containing linseed oil. Prog. Org. Coat. 2012, 75, 292-300. [CrossRef] 
27. Leal, D.A.; Riegel-Vidotti, I.C.; Ferreira, M.G.S.; Marino, C.E.B. Smart coating based on double stimuli-responsive microcapsules containing linseed oil and benzotriazole for active corrosion protection. Corros. Sci. 2018, 130, 56-63. [CrossRef]

28. Lang, S.; Zhou, Q. Synthesis and characterization of poly(urea-formaldehyde) microcapsules containing linseed oil for self-healing coating development. Prog. Org. Coat. 2017, 105, 99-110. [CrossRef]

29. Kozdrach, R.; Skowronski, J. The application of polyvinylpyrrolidone as a modifier of tribological properties of lubricating greases based on linseed oil. J. Tribol. 2018, 140, 1-7. [CrossRef]

30. Li, H.; Cui, Y.; Li, Z.; Zhu, Y.; Wang, H. Fabrication of microcapsules containing dual-functional tung oil and properties suitable for self-healing and self-lubricating coatings. Prog. Org. Coat. 2018, 115, 164-171. [CrossRef]

31. Li, H.; Cui, Y.; Wang, H.; Zhu, Y.; Wang, B. Preparation and application of polysulfone microcapsules containing tung oil in self-healing and self-lubricating epoxy coating. Coll. Surf. A Physicochem. Eng. Asp. 2017, 518, 181-187. [CrossRef]

32. Szabó, T.; Molnár-Nagy, L.; Bognár, J.; Nyikos, L.; Telegdi, J. Self-healing microcapsules and slow release microspheres in paints. Prog. Org. Coat. 2011, 72, 52-57. [CrossRef]

33. Liu, M.; Wang, Y.; Wu, Y.; Wan, H. Hydrolysis and recycling of urea formaldehyde resin residues. J. Hazard. Mater. 2018, 355, 96-103. [CrossRef] [PubMed]

34. Wang, H.; Zhou, Q. Evaluation and failure analysis of linseed oil encapsulated self-healing anticorrosive coating. Prog. Org. Coat. 2018, 118, 108-115. [CrossRef]

35. Di Credico, B.; Levi, M.; Turri, S. An efficient method for the output of new self-repairing materials through a reactive isocyanate encapsulation. Eur. Polym. J. 2013, 49, 2467-2476. [CrossRef]

36. Fan, C.; Zhou, X. Effect of emulsifier on poly(urea-formaldehyde) microencapsulation of tetrachloroethylene. Polym. Bull. 2011, 67, 15-27. [CrossRef]

37. Çömlekçi, G.K.; Ulutan, S. Encapsulation of linseed oil and linseed oil based alkyd resin by urea formaldehyde shell for self-healing systems. Prog. Org. Coat. 2018, 121, 190-200. [CrossRef]

38. de Cademartori, P.H.G.; Artner, M.A.; de Freitas, R.A.; Magalhaes, W.L.E. Alumina nanoparticles as formaldehyde scavenger for urea-formaldehyde resin: Rheological and in-situ cure performance. Compos. Part B 2019, 176, 107281. [CrossRef]

39. Dorieh, A.; Mahmoodi, N.O.; Mamaghani, M.; Pizzi, A.; Zeydib, M.M.; Moslemid, A. New insight into the use of latent catalysts for the synthesis of urea formaldehyde adhesives and the mechanical properties of medium density fiberboards bonded with them. Eur. Polym. J. 2019, 112, 195-205. [CrossRef]

40. Roumeli, E.; Papadopoulou, E.; Pavlidou, E.; Vourlias, G.; Bikiarisc, D.; Paraskevopoulosa, K.M.; Chrissafisa, K. Synthesis, characterization and thermal analysis of urea-formaldehyde/nanoSiO ${ }_{2}$ resins. Thermochim. Acta 2012, 527, 33-39. [CrossRef]

41. Jorcin, J.; Orazem, M.E.; Nadine, P.; Tribollet, B. CPE analysis by local electrochemical impedance spectroscopy. Electrochim. Acta 2006, 51, 1473-1479. [CrossRef]

(C) 2019 by the authors. Licensee MDPI, Basel, Switzerland. This article is an open access article distributed under the terms and conditions of the Creative Commons Attribution (CC BY) license (http://creativecommons.org/licenses/by/4.0/). 